\title{
POLARISATION DANS LA RÉSONANCE PARAMAGNÉTIQUE ÉLECTRONIQUE DE ZnTe/Fe ${ }^{+++}$
}

\author{
B. CLERJAUd et A. CHEVY \\ Laboratoire de Luminescence II, Faculté des Sciences de Paris \\ Equipe de Recherches Associée au C. N. R. S.
}

\begin{abstract}
Résumé. - Le champ cristallin introduit une absorption de la composante circulaire anormale du champ hyperfréquence, ce qui se traduit dans un spectre de résonance paramagnétique électronique par une variation angulaire de l'intensité des raies. Cet effet est particulièrement marqué dans le cas du $\mathrm{ZnTe} / \mathrm{Fe}^{+++}$où le paramètre de champ cristallin cubique est exceptionnellement important $\left(a=-0,2613 \mathrm{~cm}^{-1}\right)$. Des effets analogues existent pour les transitions ( interdites ) $\Delta M=2,3$, $4,5$.
\end{abstract}

Abstract. - The crystal field introduces an absorption of the abnormal circular component of the microwave which is to be expressed in an E. P. R. spectrum by an angular variation of the intensities of the lines. This effect is especially marked in the case of $\mathrm{ZnTe} / \mathrm{Fe}^{+++}$where the cubic crystal field parameter is exceptionally important $\left(a=-0,2613 \mathrm{~cm}^{-1}\right)$. Similar effects exist for the ( forbidden ) transitions $\Delta M=2,3,4,5$.

Dans une expérience de Résonance Paramagnétique Electronique (R. P. E.) sur des ions libres, l'absorption de l'onde hyperfréquence se fait uniquement grâce au terme $\frac{1}{2} g \beta H_{1}^{-} S^{+}$où $H_{1}$ représente le champ magnétique hyperfréquence et $S^{+}=S_{X}+i S_{Y}$. Il y a donc absorption d'une seule composante circulaire du champ hyperfréquence. Nous allons voir que le champ cristallin introduit une absorption de l'autre composante circulaire, et nous discuterons les conséquences de cet effet. Nous nous placerons dans le cas particulier d'un ion $3 \mathrm{~d}^{5}$ dans un site de symétrie $T_{d}$.

I. Hamiltonien de spin. - L'hamiltonien de spin à employer pour les ions $3 \mathrm{~d}^{5}$, sans spin nucléaire, dans un site de symétrie $T_{d}$ est :

$$
\begin{aligned}
\mathscr{H}=g \beta \mathbf{S H}+\frac{a}{6}\left[S_{x}^{4}+S_{y}^{4}+S_{z}^{4}-\right. & \\
& \left.\quad-\frac{1}{5} S(S+1)\left(3 S^{2}+3 S-1\right)\right]
\end{aligned}
$$

$x y z$ représentent les axes d'ordre 4 du cristal. Nous négligeons l'anisotropie du tenseur $g$, le terme décrivant un ordre supérieur de l'interaction entre les électrons $3 \mathrm{~d}$ et le champ magnétique.

Cet hamiltonien est équivalent à l'opérateur :

$$
\mathscr{H}=g \beta \mathbf{S H}+\frac{8}{120} \sqrt{\frac{4 \pi}{9}} a\left[Y_{4}^{0}+\sqrt{\frac{5}{14}}\left(Y_{4}^{4}+Y_{4}^{-4}\right)\right] .
$$

En général, le terme $g \beta$ SH prédomine, nous écrivons donc l'hamiltonien dans un repère dans lequel ce terme est diagonal, c'est-à-dire dans un repère où l'axe de quantification $Z$ est colinéaire au champ magnétique. Pour cela nous employons les formules de rotation des harmoniques sphériques [1]

$$
Y_{l}^{m}(\omega)=\sum_{m^{\prime}=-l}^{+l} Y_{l}^{m^{\prime}}(\Omega) R_{m^{\prime} m}^{l}(\alpha \theta \gamma)
$$

$\omega$ et $\Omega$ représentent les coordonnées angulaires sphériques dans l'ancien et le nouveau repère ; $\alpha, \theta, \gamma$ sont les angles d'Euler permettant de passer du nouveau repère à l'ancien repère.

$$
R_{m^{\prime} m}^{l}(\alpha \theta \gamma)=\mathrm{e}^{-i \alpha m^{\prime}} r_{m^{\prime} m}^{l}(\theta) \mathrm{e}^{-i \gamma m}
$$

$r_{m^{\prime} m}^{l}(\theta)$ est donné par la formule de Wigner :

$r_{m^{\prime} m}^{l}(\theta)=$

$$
\begin{gathered}
=\sum_{t}(-)^{t}-\frac{\sqrt{\left(l+m^{\prime}\right) !\left(l-m^{\prime}\right) !(l+m) !(l-m) !}}{\left(l+m^{\prime}-t\right) !(l-m-t) ! t !\left(t-m^{\prime}+m\right) !} \times \\
\times\left(\cos \frac{\theta}{2}\right)^{2 l+m^{\prime}-m-2 t}\left(\sin \frac{\theta}{2}\right)^{2 t-m^{\prime}+m}
\end{gathered}
$$

$\sum_{t}$ indique une sommation sur toutes les valeurs entières de $t$ pour lesquelles les factorielles ont un sens, c'est-àdire pour lesquelles les arguments des factorielles sont positifs ou nuls.

Si le champ magnétique est dans le plan (1110) du cristal, on a $\alpha=0 ; \theta=$ angle entre $H$ et l'axe [001] ; $\gamma=\pi / 4$ et l'hamiltonien s'écrit alors :

$$
\mathscr{H}=g \beta S_{Z} H+\sum_{i=-4}^{+4} V_{4}^{i}
$$


avec :

$$
\begin{aligned}
V_{4}^{0}=\frac{a}{480} & {\left[35 S_{Z}^{4}-\{30 S(S+1)-25\} S_{Z}^{2}+\right.} \\
& \left.+3 S^{2}(S+1)^{2}-6 S(S+1)\right] \times \\
& \times\left[15 \cos ^{4} \theta-10 \cos ^{2} \theta-1\right] . \\
V_{4}^{ \pm 1}=\frac{a}{12}\{ & {\left[7 S_{Z}^{3}-[3 S(S+1)+1] S_{Z}\right] S^{ \pm}+} \\
& \left.+S^{ \pm}\left[7 S_{Z}^{3}-[3 S(S+1)+1] S_{Z}\right]\right\} \times \\
& \times\left\{\cos \theta \sin \theta\left(3 \cos ^{2} \theta-1\right)\right\} . \\
V_{4}^{ \pm 2}=\frac{a}{96}\{ & {\left[7 S_{Z}^{2}-S(S+1)-5\right] S^{ \pm 2}+} \\
& \left.+S^{ \pm 2}\left[7 S_{Z}^{2}-S(S+1)-5\right]\right\} \times \\
& \times\left\{\sin ^{2} \theta\left(3 \cos ^{2} \theta-1\right)\right\} . \\
V_{4}^{ \pm 3}=\frac{a}{48}\{ & \left.S_{Z} S^{ \pm 3}+S^{ \pm 3} S_{Z}\right\} \times \\
& \times\left\{\sin ^{ \pm} \theta \cos ^{ \pm} \theta\left(5-3 \cos ^{2} \theta\right)\right\} . \\
V_{4}^{ \pm 4}=\frac{a}{192} & S^{ \pm 4}\left(3 \cos ^{2} \theta-1\right)\left(\cos ^{2} \theta-3\right) .
\end{aligned}
$$

II. Absorption des deux composantes circulaires. L'hamiltonien perturbateur qui induit les transitions est :

$$
\mathcal{H}_{1}^{\prime}=\mathcal{H}_{1} \cos \omega t=g \beta \mathbf{S} \cdot \mathbf{H}_{1} \cos \omega t .
$$

La probabilité de transition par unité de temps entre les niveaux $M^{\prime}$ et $M$ est, à énergie constante, donnée par :

$$
W_{M^{\prime} M}=\frac{\pi^{2}}{h^{2}} g(v)\left|<\psi_{M}\right| \mathcal{H}_{1}\left|\psi_{M^{\prime}}>\right|^{2}
$$

Si le champ $H_{1}$ est normal au champ magnétique statique, cette probabilité de transition devient :

$$
W_{M^{\prime} M}=\frac{\pi^{2}}{4 h^{2}} g(v)\left|<\psi_{M}\right| H_{1}^{+} S^{-}+H_{1}^{-} S^{+}\left|\psi_{M^{\prime}}>\right|^{2}
$$

où

$$
\begin{aligned}
& H_{1}^{+}=H_{1 X}+i H_{1 Y} \\
& H_{1}^{-}=H_{1 X}-i H_{1 Y} .
\end{aligned}
$$

Nous calculons des quantités $p_{+}$et $p_{-}$dont le carré est proportionnel à la probabilité de transition :

$$
\begin{aligned}
& p_{+}=\left\langle\psi_{M}\left|S^{+}\right| \psi_{M^{\prime}}\right\rangle \\
& p_{-}=\left\langle\psi_{M}\left|S^{-}\right| \psi_{M^{\prime}}\right\rangle .
\end{aligned}
$$

Nous nous limitons à un développement au premier ordre en perturbation pour les fonctions d'ondes:

$\left.\left|\psi_{M}\right\rangle=\left|M>+\sum_{M^{\prime} \neq M} \frac{<M^{\prime}|\mathscr{H}| M>}{E_{M}-E_{M^{\prime}}}-\right| M^{\prime}\right\rangle$

les $\mid M>$ étant les états propres communs à $S_{Z}$ et $S^{2}$.
Pour les transitions $\Delta M=1, p_{+}$est donné par :

$$
\begin{aligned}
p_{+}=\left\langle M\left|S^{+}\right| M-1\right. & >= \\
& =\sqrt{S(S+1)-M(M-1)}
\end{aligned}
$$

l'intensité des raies ne dépend pas de l'orientation.

Pour obtenir $p_{-}$, il faudra tenir compte des termes du premier ordre

$$
\begin{aligned}
& p_{-:}=\frac{<M+1\left|V_{4}^{2}\right| M-1>}{-2 g \beta H}<M\left|S^{-}\right| M+1>+ \\
& +\frac{<M-2\left|V_{4}^{-2}\right| M>}{2 g \beta H}<M-2\left|S^{-}\right| M-1>
\end{aligned}
$$

ce qui donne tous calculs effectués :

$$
\begin{aligned}
& p_{-}=\frac{a}{96 g \beta H} \sin ^{2} \theta\left(3 \cos ^{2} \theta-1\right) \times \\
& \times \sqrt{S(S+1)-M(M-1)} \times \\
& \times\left[7\left(6 M^{3}-9 M^{2}+11 M-4\right)-\right. \\
& -2(M-1)\{9 S(S+1)-10\}]
\end{aligned}
$$

cette amplitude est nulle pour la transition $-\frac{1}{2} \rightarrow \frac{1}{2}$ mais différente de zéro pour les autres transitions; elle varie avec l'orientation du cristal.

III. Conséquence en R. P. E. « classique ». - Lorsqu'on travaille avec une onde hyperfréquence polarisée rectilignement, la probabilité de transition est proportionnelle à :

$$
\left|H_{1}^{-} p_{+}+H_{1}^{+} p_{-}\right|^{2} \text {. }
$$

On a d'autre part :

$$
\begin{aligned}
& H_{1 X}=H_{1} \cos \varphi \\
& H_{1 Y}=H_{1} \sin \varphi .
\end{aligned}
$$

$\varphi$ étant l'angle entre $H_{1}$ et le plan (110). La probabilité de transition est donc proportionnelle à :

$$
f=\left(p_{+}+p_{-}\right)^{2} \cos ^{2} \varphi+\left(p_{+}-p_{-}\right)^{2} \sin ^{2} \varphi
$$

ce qui peut se mettre sous la forme :

$$
f=\left(p_{+}+p_{-} \cos 2 \varphi\right)^{2}+p_{-}^{2} \sin ^{2} 2 \varphi
$$

on voit donc que l'intensité des raies variera :

- avec l'orientation du cristal,

- avec la direction du champ magnétique hyperfréquence.

IV. Transitions interdites. - Des transitions $\Delta M \neq 1$ sont permises à cause des termes non diagonaux de l'hamiltonien.

a) $\Delta M=2$. - L'absorption de la composante circulaire normale est assurée grâce aux termes $V_{4}^{1}$ et $V_{4}^{-1}$

$$
p_{+} \sim \cos \theta \sin \theta\left(3 \cos ^{2} \theta-1\right)
$$

l'absorption de la composante circulaire anormale s'effectue grâce aux termes $V_{4}^{3}$ et $V_{4}^{-3}$

$$
p_{-} \sim \sin \theta \cos \theta\left(5-3 \cos ^{2} \theta\right) \text {. }
$$


b) $\Delta M=3 .-$ L'absorption de la composante normale se fait grâce aux termes $V_{4}^{2}$ et $V_{4}^{-2}$

$$
p_{+} \sim \sin ^{2} \theta\left(3 \cos ^{2} \theta-1\right)
$$

l'absorption de la composante anormale est autorisée grâce à $V_{4}^{4}$ et $V_{4}^{-4}$

$$
p_{-} \sim\left(3 \cos ^{2} \theta-1\right)\left(\cos ^{2} \theta-3\right) \text {. }
$$

c) $\Delta M=4$. - L'absorption de la composante normale est permise grâce à $V_{4}^{3}$ et $V_{4}^{-3}$

$$
p_{+} \sim \sin \theta \cos \theta\left(5-3 \cos ^{2} \theta\right)
$$

l'absorption de la composante anormale est interdite. $p_{-}=0$.

d) $\Delta M=5$. - L'absorption de la composante normale est autorisée grâce à $V_{4}^{4}$ et $V_{4}^{-4}$

$$
p_{+} \sim\left(3 \cos ^{2} \theta-1\right)\left(\cos ^{2} \theta-3\right)
$$

l'absorption de la composante anormale est interdite. $p_{-}=0$.

V. Application au cas de $\mathrm{ZnTe} / \mathrm{Fe}^{+++}$. - Ces effets sont particulièrement sensibles dans le cas du $\mathrm{ZnTe} / \mathrm{Fe}^{+++}$où le paramètre de champ cubique déterminé par J.-C. Hensel [2] est très important :

$$
a=-0,2613 \mathrm{~cm}^{-1} \text {. }
$$

Pour que notre traitement par perturbations reste valable, nous évaluons la grandeur de ces effets en bande $\mathrm{Ka}(v=35 \mathrm{GHz})$. Nous étudions le cas où le champ magnétique est colinéaire à l'axe [110] du cristal, c'est-à-dire $\theta=\pi / 2$. L'absorption ordinaire donne pour le spectre principal les intensités observées dans les cas où a est faible : $8: 5: 9: 5: 8$ (Fig. 1a); l'absorption de l'autre composante circulaire donne un spectre avec les intensités suivantes : $0,15: 0,6: 0$ : $0,6: 0,15$ (Fig. 1b).

En R.P. E. où l'onde est polarisée rectilignement, les raies $\frac{3}{2} \rightarrow \frac{5}{2}$ et $-\frac{5}{2} \rightarrow-\frac{3}{2}$ n'ont plus les mêmes intensités car les amplitudes $p_{-}$sont de signes contraires. Il en est de même pour les transitions $\frac{1}{2} \rightarrow \frac{3}{2}$ et $-\frac{3}{2} \rightarrow-\frac{1}{2}$. Les intensités sont proportionnelles à : $10,4: 2,1: 9: 9,2: 5,9$ pour $H_{1} \perp(1 \overline{1} 0)$ (Fig. $1 c$ ) et $5,9: 9,2: 9: 2,1: 10,4$ pour $H_{1} / /[001]$ (Fig. 1d).

Conclusion. - Cet effet sensible dans le cas du $\mathrm{ZnTe} / \mathrm{Fe}^{+++}$en bande $\mathrm{Ka}$ doit devenir très important en bande $X:$ l'absorption des deux ondes circulaires doit être du même ordre de grandeur ; un calcul de perturbations n'est alors plus valable et il faudra diagonaliser l'hamiltonien à l'aide d'un ordinateur.
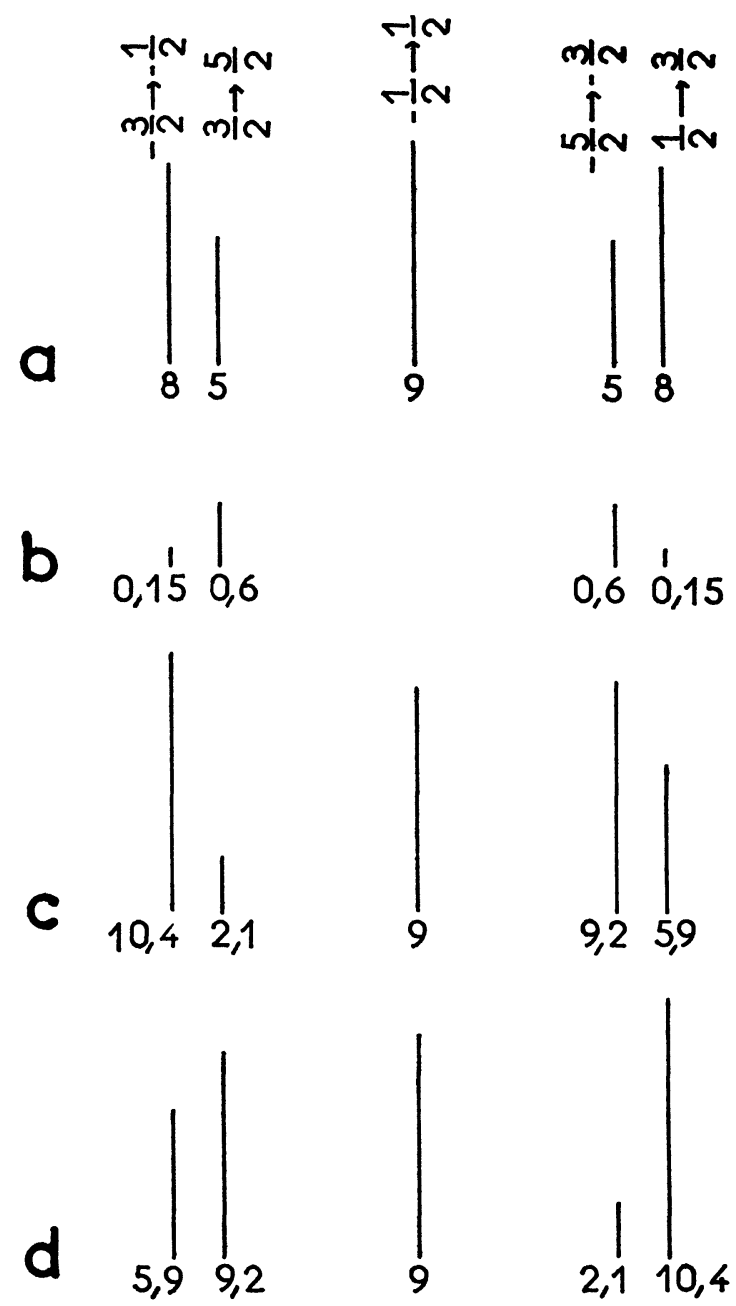

Fig. 1. $-\mathrm{ZnTe} / \mathrm{Fe}^{+++}$: Intensité des raies pour $H / /[110]$ à $v=35 \mathrm{GHz}$. a) Onde polarisée circulairement dans le sens normal ; $b$ ) Onde polarisée circulairement dans le sens anormal; c) Onde polarisée rectilignement : $\left.H_{1} \perp(1 \overline{10}) ; d\right)$ Onde polarisée rectilignement : $H_{1} / /[001]$.

Ce phénomène est gênant pour déterminer le signe du paramètre de champ cristallin ; pour cela on compare habituellement les intensités des raies $\frac{3}{2} \rightarrow \frac{5}{2}$ et $-\frac{5}{2} \rightarrow-\frac{3}{2}$ d'une part, et $\frac{1}{2} \rightarrow \frac{3}{2}$ et $-\frac{3}{2} \rightarrow-\frac{1}{2}$ d'autre part, à basse température, car les niveaux les plus bas sont les plus peuplés ; cette méthode devient inopérante dans le cas de forts champs cristallins, et il faudrait opérer en onde polarisée circulairement.

Il sera intéressant de mesurer le paramètre a dans $\mathrm{CdTe} / \mathrm{Fe}^{+++}$pour vérifier la théorie de G. H. Azarbayejani [3]. Ce paramètre sera vraisemblablement important et la détermination du signe serait certainement facilitée par une expérience en onde polarisée circulairement.

\section{Bibliographie}

[1] Messiah (A.), Mécanique quantique ; Ed. Dunod, 1960. [3] Azarbayejani (G. H.), « An M. O. Theoiy of the cubic [2] Hensel (J. C.), Bull. Am. Phys. Soc., 1964, 9, 244. field splitting of the S-state ions in II-VI compounds of $T_{d}$ symmetry ). The University of Michigan, Ph. D., 1966. 